\title{
Retraction Note to: Prognostic investigations of B7-H1 and B7-H4 expression levels as independent predictor markers of renal cell carcinoma
}

\author{
Hamid Reza Safaei ${ }^{1}$ Ayoob Rostamzadeh ${ }^{2} \cdot$ Omid Rahmani $^{3} \cdot$ Mohsen Mohammadi $^{4}$. \\ Omar Ghaderi ${ }^{5} \cdot$ Hamid Yahaghi $^{6} \cdot$ Koroosh Ahmadi $^{7}$
}

Published online: 9 November 2016

(C) International Society of Oncology and BioMarkers (ISOBM) 2016

Retraction Note to: Tumor Biol (2016) 37:7583-7587

DOI 10.1007/s13277-015-4652-Z

This article has been retracted at the request of the Editor-inChief, the International Society of Oncology and BioMarkers (ISOBM) and the Publisher per the Committee on Publication Ethics guidelines. The article shows evidence of irregularities in authorship and in the materials and methods during the submission process, and there is strong reason to believe that the peer review process was compromised.

As such the validity of the content of this article cannot be verified.

The online version of the original article can be found at http://dx.doi. org/10.1007/s13277-015-4652-z.

Koroosh Ahmadi

ahmadik@mums.ac.ir

1 Department of Pediatric Nephrology, AJA University of Medical

Sciences, Tehran, Iran

2 Department of Anatomical Sciences, Faculty of Medicine, Shahrekord University of Medical Sciences, Shahrekord, Iran

3 Department of Pathology, Be'sat Hospital, AJA University of Medical Sciences, Tehran, Iran

4 Department of Pharmaceutical Biotechnology, Faculty of Pharmacy, Lorestan University of Medical Sciences, Khorramabad, Iran

5 Department of Pharmaceutical Biotechnology, Tehran University of Medical Sciences, Tehran, Iran

6 Department of Molecular Biology, Baqiyatallah University of Medical Sciences, Tehran, Iran

7 Department of Emergency Medicine, Alborz University of Medical Sciences, Karaj, Iran 